\title{
Educación para la Ciudadanía Global (ECG): Comprendiendo lo internacional a través de lo local
}

\author{
Ánxela Bugallo-Rodríguez \& María Cristina \\ Naya-Riveiro
}

Resumen:

En la actualidad, hablamos de Educación para la Ciudadanía Global como un tema transversal básico para contribuir a dar respuesta a los principales problemas mundiales de la humanidad. Su carácter transformador nos obliga a buscar formas de aprendizaje que generen conciencia crítica, haciéndonos responsables y activos en la búsqueda de un mundo más equitativo e igualitario. Un objetivo tan ambicioso precisa de nuevas fórmulas que aseguren su implantación, más allá de las acciones puntuales que se vienen realizando, a través de procesos interactivos y participativos que busquen una formación integral. Para conseguirlo el presente trabajo propone abordar esta temática desde una colaboración amplia, desde el profesorado de Educación Infantil hasta el universitario. El resultado es un proyecto integral de centro que trata la ECG en contexto, partiendo de un tema local para alcanzar objetivos globales. El procedimiento propuesto se puede proyectar a cualquier centro educativo, tomando como referencia un tema de interés en el desarrollo socioeconómico de la zona. Nos permite educar, sensibilizar e investigar sobre cuestiones locales y globales, para así poder decidir.

Palabras clave:

educación para la ciudadanía global; innovación educativa; programa colaborativo. 


\title{
Global citizenship education (GCE): understanding the international topic across the local topic
}

\begin{abstract}
Nowadays we talk about Global Citizenship Education as a basic cross-curricular approach that contributes to responding to the major world problems of humanity. Its transformative character is based on a form of learning that generates critical awareness, preparing responsible people who actively seek a more equitable and egalitarian world. This ambitious aim requires new methods that ensure a better implementation than the isolated actions that are being carried out, through interactive and participatory processes that aim to provide a holistic education. In order to achieve that, the present work proposes to approach this subject from wide collaboration, from the teaching staff of Early Childhood to University. The result is a comprehensive project which implements GCE in context, starting from a local topic to address global issues. The procedure is proposed for a later projection in any school centre, based on a central topic related to socioeconomic development of the area as a point of reference. This allows us to educate, raise awareness and research both local and global issues, in order to facilitate decision-making.
\end{abstract}

Key words: global citizenship education; educational innovation; collaborative program.

\section{Éducation pour la citoyenneté globale: compréhension de l'international à travers du local}

Résumé: Aujourd'hui, nous parlons de l'éducation pour la citoyenneté mondiale en tant que thème transversal de base pour aider à répondre aux grands problèmes mondiaux de l'humanité. Son caractère transformateur nous force à chercher des moyens d'apprentissage qui génèrent la conscience critique, nous rendent responsables et actifs dans la recherche d'un monde plus équitable et égalitaire. Un objectif aussi ambitieux nécessite de nouvelles formules, qui assurent une mise en œuvre au-delà des actions spécifiques en cours, grâce à des processus interactifs et participatifs qui cherchent à former complètement. Pour y parvenir, le présent travail propose d'approcher ce sujet d'une large collaboration, du personnel enseignant de l'éducation infantile à l'université. Le résultat est un projet complet du centre qui est l'ECG dans le contexte, d'un problème local pour atteindre les objectifs mondiaux. La procédure est proposée pour une projection ultérieure dans n'importe quel centre éducatif, en prenant comme référence un sujet d'intérêt dans le développement socio-économique de la région. Cela nous permet d'éduquer, de sensibiliser et de faire des recherches sur les problèmes locaux et mondiaux, afin que nous puissions décider.

Mots clés: éducation à la citoyenneté mondiale; i'innovation éducative; programme collaboratif.

\section{Educação para a cidadania global: compreendendo o internacional pelo local}

Resumo: Atualmente, falamos da Educação para a Cidadania Global como um tema transversal básico para responder aos principais problemas mundiais da humanidade. Sua natureza transformadora obriga a pesquisar formas de aprendizagem que gerem consciência crítica, tornando-nos responsáveis e ativos na procura de um mundo mais equitativo e igualitário. A realização de um objectivo tão ambicioso exige novas fórmulas, que assegurem uma implementação além das acções específicas que estão sendo realizadas, através de processos interativos e participativos na vontade de uma formação integral. Para obtê-lo, o presente trabalho propõe focar esse assunto de uma colaboração ampla, desde o corpo docente da educação infantil até a universidade. O resultado é um projeto abrangente de Centro, que trata o ECG em contexto, a partir de um tema local para alcançar objetivos globais. O procedimento é proposto para uma projeção posterior em qualquer centro educacional, tomando como referência um tema de interesse no desenvolvimento socioeconômico da área. Isso nos permite educar, conscientizar e pesquisar sobre questões locais e globais, para que possamos decidir.

Palavras-chave: educação para a cidadania global; inovação educacional; programa colaborativo. 


\section{La Educación para la ciudadanía global: de la Unesco a la escuela}

Globalización, ciudadanía y desarrollo son hechos clave del debate actual, y la educación debe reflejarlo y ayudar a la comprensión del impacto de esa mundialización en el contexto local. La necesidad de incorporar estos temas ya fue planteada oficialmente en 1974, cuando un organismo internacional como la Unesco instaba a pensar la educación como un medio para contribuir a resolver los problemas que condicionan la supervivencia y el bienestar de la humanidad, la desigualdad, la injusticia, las relaciones mundiales basadas en el uso de la fuerza y a caminar hacia medidas de cooperación internacional que faciliten su solución (Unesco, 1974).

Desde ese objetivo, enmarcado en la Educación para el Desarrollo (EpD) se ha avanzado en las dos últimas décadas y diversos autores, en estos años, han creado una pasarela desde ésta hacia la Educación para la Ciudadanía Global (ECG).

Autores como Ortega (2007) y Aguado (2011) hablan de EpD como una educación que intenta relacionar lo local con lo global, resaltando la interdependencia de ambas dimensiones; como una educación integral, basada en mostrar los problemas y sus causas; una educación que toma los valores morales de la Declaración Universal de los Derechos Humanos como horizonte axiológico. De esta forma, la estrategia de la EpD pretende promover una Ciudadanía Global.

Davies, Evans y Reid (2005) han propuesto que sería útil ir más allá de esas viejas barreras que han separado Educación Global y Educación para la Ciudadanía. La ECG no sería simplemente un híbrido de ambas, sino un nuevo tipo de educación que ofrece la oportunidad de transformar el contenido de los programas de estudio, el entorno de aprendizaje y las prácticas didácticas.

Entramos en una época en la que ya se está utilizando el nuevo término de "Educación para la Ciudadanía Global/Mundial" (Unesco, 2015, 2016) que "aspira a ser un factor de transformación, inculcando los conocimientos, las habilidades, los valores y las actitudes que los educandos necesitan para poder contribuir a un mundo más inclusivo, justo y pacífico" (Unesco, 2015, p. 15). Destaca, así, una función especial de la educación, aquella relacionada con la formación de la ciudadanía en un mundo interconectado e interdependiente, impulsado por los múltiples procesos asociados con la globalización (Tawil, 2013).

El concepto de ECG tiene diferentes interpretaciones como muestran los estudios de Su, Bullivant y Holt (2013). El modelo educativo aquí escogido es de carácter humanista y global (Paz, 2007) y propone una educación integral, con una perspectiva global de la justicia y de la solidaridad. Apuesta por una enseñanza orientada al compromiso y a la acción, que aliente al alumnado a responsabilizarse, a tomar decisiones y a mejorar su entorno. Razones por las que a este marco educativo se vinculan otros grandes objetivos actuales, como son el desarrollo sostenible y el consumo responsable. 
En general, la entrada de la ECG en el curriculum español se produce a través de la promoción de la competencia social y cívica y, aunque se supone que será tratada de forma transversal por las diferentes materias, raramente aparece incluida y acaba funcionando en actividades aisladas. Opinamos que su tratamiento no puede ser puntual, ni abordarse desde unas pocas áreas, y, al igual que en otros estados como Reino Unido (Su, Bullivant \& Holt, 2013), se debe incorporar transversalmente al curriculum oficial para tratarse a nivel de centro. De hecho, en el presente trabajo hemos avanzado hacia un proyecto integral de centro, implicando a todos los niveles educativos en la elaboración de un programa de ECG.

Pero hemos ido un paso más allá y buscamos redefinir el papel de la universidad, y de sus especialistas, como agentes activos para construir escenarios alternativos. No sólo en su propio ámbito sino extendiendo su acción, promoviendo proyectos conjuntos con actores de la sociedad civil (Leite \& Beltrán, 2012) y con profesorado de otros niveles educativos, implicándose directamente con una comunidad local.

\section{Objetivo: un proyecto integral de centro para trabajar la ECG}

El trabajo aborda las posibilidades de introducir la ECG a partir de un proyecto de investigación colaborativa de amplio rango, en el que participa profesorado de un Colegio Público Integrado, especialistas universitarios de Didáctica y miembros de una Organización no Gubernamental centrada en temas de Educación para el Desarrollo (ONGD).

En cuanto a los contenidos se ha escapado de una visión simplista de la ECG, que considere que la mera inclusión de contenidos internacionales pueda conseguir el diseño de este tipo de programas (Davies, Evans \& Reid, 2005). Y la apuesta es por el aprendizaje de la dimensión mundial de los temas locales, presentes en todas las vidas y comunidades (Brownlie, 2001). Porque, debido a los vínculos internacionales, una acción local, como en este caso la producción, venta y consumo de un alimento, podría tener un impacto más amplio (Davies, 2008).

El objetivo es introducir la ECG transversalmente en un centro, incrementando la involucración del profesorado, atendiendo a sus intereses y necesidades y a las de su alumnado. ¿Quiere esto decir que nos planteamos un objetivo para un centro específico? Nada más lejos, pues lo que se busca es el desarrollo de marcos, procedimientos e interpretaciones útiles para investigadores y docentes, de forma que el proceso de elaboración de este programa sea trasladable a cualquier centro.

Las preguntas de investigación planteadas fueron las siguientes:

- ¿Cómo se puede incorporar transversalmente la ECG en la práctica curricular de un centro educativo concreto?

- ¿Qué tipo de propuestas didácticas debemos diseñar para incluir la ECG en la práctica docente del centro? 
- ¿Cuál es el papel que puede tener la universidad en la extensión de la ECG?

Estos interrogantes permitirán conocer cómo un programa de esta naturaleza puede favorecer la mejora de la intervención, a partir de un proceso colaborativo (Barkeley, 2007) e innovador de diseño. La detección de áreas prioritarias a trabajar con el alumnado y el profesorado, ayudará a motivarlos para participar activamente en un modelo de desarrollo humano colectivo, a través de la comprensión crítica de la realidad local (Fricke, Gathercole \& Skinner, 2015).

\section{Una metodología innovadora y compleja para la ECG: Colaboración de todos los niveles educativos}

El programa realizado es el resultado de las dos fases (2015-16 y 2016-17) de un proyecto piloto desarrollado en un centro público de Galicia, que ha implicado a una gran parte del personal docente del propio centro y a numerosos investigadores de la universidad.

Para su desarrollo se ha optado por una estrategia de investigación cualitativa, concretamente a través de la investigación-acción colaborativa, elegida por dos motivos. Primero, es la más apropiada para comprender el significado que los sujetos otorgan a las experiencias sociales y educativas que protagonizan. Segundo, posibilita el avance en la educación orientada a las decisiones (Imbernón, 2002), aspecto clave para el tema.

El proyecto se beneficia del profesorado del centro, quien conoce bien a los estudiantes, la localidad y el ambiente, y los incorpora a una concepción unificada de la práctica reflexiva educativa (Membiela, 2002). Se ha pretendido así que el profesorado sea protagonista de la investigación, pues son ellos los que incorporan las temáticas de la ECG dentro de sus materias.

El principal propósito es comprender las experiencias sociales en sus escenarios naturales, en este caso centrándonos en un tema clave de la economía local, y generar un proceso de auto-transformación que promueva cambios en la práctica (Kemmis \& McTaggart, 1988).

El proceso de diseño del programa ha sido complejo y ha incluido aspectos innovadores que exponemos a continuación:

a. La universidad como agente del cambio

El estudio tiene su origen en la decisión de un grupo de investigación universitario de desarrollar un proyecto colaborativo como fórmula para afrontar temáticas transversales de esta índole que, normalmente, quedan limitadas a actividades puntuales realizadas por organizaciones no gubernamentales, o por docentes de forma individual. El grupo de especialistas está formado por profesorado de las áreas de Didáctica General y Específicas (Didáctica de las Ciencias Experimentales, de las Ciencias Sociales, de 
Matemáticas, de la Lengua y Literatura y de Lenguas Extranjeras) de la Universidad de A Coruña.

b. Selección del centro educativo

Se alcanzó un consenso sobre los criterios que debía reunir un centro educativo para ser centro piloto. De entre siete centros preseleccionados, se escogió el C.P.I. Plurilingüe "Virxe da Cela" de Monfero, un colegio público donde se habían desarrollado, con anterioridad, experiencias en EpD y donde ya existía una cultura de la experimentación en torno a diversas áreas del curriculum.

c. Creación del grupo de trabajo mixto

Se constituyó un único gran grupo de trabajo, con los docentes del centro dispuestos a participar y con el grupo de especialistas universitarios que actúan como asesores y orientadores. El profesorado pertenecía a todos los departamentos del centro educativo, su experiencia docente oscilaba entre uno y veinte años y su formación disciplinar era la correspondiente a su campo.

d. Diseño del programa integral "en contexto"

El grupo de trabajo debatió sobre cuál podría ser la muestra de alumnado y decidió hacerlo en las tres etapas educativas (Infantil, Primaria y Secundaria), dándole así al programa un carácter integral. Siguiendo las demandas del centro, los tres planes didácticos del programa abordan los contenidos de la ECG en relación con una temática del contexto local, el sector lácteo-ganadero, base económica de una parte importante de la población de la zona rural en la que está situado este centro.

\section{Un programa de ECG de lo local a lo internacional}

Se ha partido así de una de las dimensiones conceptuales básicas de la ECG (Unesco, 2015), para conseguir un resultado de aprendizaje en el que los educandos aprenden a conocer y comprender los problemas locales, nacionales (situación del sector lácteo) y mundiales, así como las interrelaciones e interdependencia de los diferentes países (comercio global).

Para profundizar en aspectos relativos al contexto conviene recordar que el proyecto se realiza en un Colegio Público Integrado, un tipo de centros creados para adaptarse a las peculiaridades de la distribución geográfica de la población. Monfero es un ayuntamiento de amplia extensión territorial $\left(172,4 \mathrm{~km}^{2}\right)$ y tendencia negativa en su demografía (2.019 habitantes), con una densidad de 12,17 habitantes por km², reflejo de la situación que vive Galicia, pues es la comunidad autónoma que lidera el descenso de población rural. Este ayuntamiento concentra la mayoría de granjas (169) de la comarca y, aunque son explotaciones de tamaño pequeño, ésta es su principal fuente económica, en fuerte declive en la actualidad. 
El proyecto de trabajo de ECG para el colegio se ha centrado en esa importante temática local: las explotaciones ganaderas y la leche como su producto principal.

El programa fue desarrollado en tres planes con los siguientes objetivos generales:

- Alcanzar aprendizajes significativos y realizar una transferencia integral a diferentes contextos y situaciones.

- Conectar los aprendizajes con la realidad global y propia a través del análisis de situaciones-problema.

- Integrar el contexto como recurso educativo de primer orden, como fuente de aprendizaje, valorando el medio natural como fuente de riqueza.

- Valorar la dedicación, el trabajo y el esfuerzo, personal y grupal, como fuente de desarrollo social.

- Fomentar la igualdad de género, la equidad y la inclusión, el respeto entre culturas, la no discriminación y la valoración de lo propio y de lo ajeno.

- Fortalecer las relaciones intergeneracionales e implicar a la comunidad educativa en los procesos de enseñanza-aprendizaje.

\section{Plan de acción 1: Explotaciones ganaderas}

Expuestos los objetivos comunes, la primera acción se centra en que el alumnado conozca y valore el trabajo del sector ganadero. La finalidad es identificar y estudiar las características de estas explotaciones, analizando diferentes tipologías, conociendo las razas vacunas que se comercializan, además de estudiar la evolución del sector en la comarca durante los últimos 70 años y su repercusión en el desarrollo.

Al estudiar el comercio de los productos del sector, se han tratado los conceptos de "precio justo" y "consumo responsable", que permitirán comprender las repercusiones del mercado en las personas y en los pueblos. Identificarán los avances tecnológicos producidos, su incidencia, su evolución, y valorarán la iniciativa y el espíritu emprendedor como vía de desarrollo económico y social. Analizarán el papel de la mujer y las barreras que deben superar personas con diversidad funcional en el medio rural y, en particular, en una explotación ganadera. Finalmente, estudiarán formas de asociacionismo como las cooperativas de producción ganadera, lo que permitirá compararlas con las productoras de café y cacao. Estos objetivos se intentan alcanzar a través de las tareas recogidas en la Tabla I. 
Tabla I: Planificación del primer plan de acción: explotaciones ganaderas.

\begin{tabular}{|c|c|c|}
\hline TAREAS & SUBTAREAS & ETAPA EDUCATIVA \\
\hline $\begin{array}{l}\text { Preparación del } \\
\text { profesorado }\end{array}$ & $\begin{array}{l}\text { Presentar el plan de acción al profesorado y trabajar } \\
\text { sus objetivos. }\end{array}$ & $\begin{array}{c}\text { Educación Infantil (El), } \\
\text { Primaria (EP) y Secundaria } \\
\text { Obligatoria (ESO). }\end{array}$ \\
\hline $\begin{array}{l}\text { Introducción } \\
\text { del programa al } \\
\text { alumnado }\end{array}$ & $\begin{array}{c}\text { Actividad motivadora inicial: encuentros sobre } \\
\text { explotaciones ganaderas. } \\
\text { Presentación de familias ganaderas del centro } \\
\text { educativo al alumnado. } \\
\text { Los alumnos deberán crear un cartel del encuentro y } \\
\text { asumir un papel de periodistas. }\end{array}$ & $\begin{array}{c}\text { El }\left(4^{\circ}, 5^{\circ} \text { y } 6^{\circ}\right) \\
\operatorname{EP}\left(1^{\circ}, 2^{\circ}, 3^{\circ}, 4^{\circ}, 5^{\circ} \text { y } 6^{\circ}\right) \\
\operatorname{ESO}\left(1^{\circ}, 2^{\circ}, 3^{\circ} \text { y } 4^{\circ}\right) .\end{array}$ \\
\hline $\begin{array}{l}\text { Diagnóstico } \\
\text { actual }\end{array}$ & $\begin{array}{c}\text { Visitar granjas de diferente tipología de producción } \\
\text { (intensiva y ecológica). } \\
\text { Preparación en el aula de la visita: } \\
\text { - Elaborar un cuaderno de campo con preguntas para } \\
\text { realizar durante la visita. } \\
\text { - Preparar recursos para optimizar la visita. }\end{array}$ & $\operatorname{EP}\left(1^{\circ}, 2^{\circ}, 3^{\circ}, 4^{\circ}, 5^{\circ}\right.$ y $\left.6^{\circ}\right)$ \\
\hline Evolución & $\begin{array}{l}\text { 1. Estudiar y analizar las consecuencias sociales, } \\
\text { económicas y culturales de la evolución de las } \\
\text { explotaciones ganaderas. } \\
\text { 2. Realizar una línea de tiempo para valorarla. } \\
\text { 3. Analizar la tecnología empleada en el sector } \\
\text { ganadero. }\end{array}$ & $\begin{array}{c}\operatorname{EP}\left(1^{\circ}, 2^{\circ}, 5^{\circ} \text { y } 6^{\circ}\right) \\
\text { ESO }\left(2^{\circ}\right) .\end{array}$ \\
\hline Sostenibilidad & $\begin{array}{l}\text { 1. Estudiar el impacto en el medio de los productos } \\
\text { empleados en las explotaciones. } \\
\text { 2. Conocer el comercio justo y su repercusión social. } \\
\text { 3. Analizar la integración e inclusión de las personas } \\
\text { en el medio rural, en particular en una explotación } \\
\text { ganadera. }\end{array}$ & $\operatorname{EP}\left(4^{\circ}, 5^{\circ}\right.$ y $\left.6^{\circ}\right)$ \\
\hline Actividad final & $\begin{array}{l}\text { Conocer diferentes razas de vacas, mediante una } \\
\text { actividad tipo "cow parade". }\end{array}$ & El, EP y ESO. \\
\hline
\end{tabular}

Fuente: Proyecto "Investigando la dimensión global de la educación para el desarrollo. Un proyecto piloto de investigación - acción colaborativa en un centro educativo de Galicia. Fase II" (PR 804A 2016/25).

\section{Plan de acción 2: La leche y sus derivados}

A continuación el profesorado ha propuesto la temática de la leche, que es el producto básico de la explotación ganadera de la zona. Cabe destacar que está muy relacionada con el consumo de los principales productos exportados desde los países del Sur, como son azúcar, cacao y café, lo que nos permitirá trabajar la relación como ciudadano global.

En este segundo plan los objetivos específicos tocan tres aspectos de la producción láctea (la elaboración, el consumo y su relación con la salud), desarrollados a través de una serie de tareas expuestas en la Tabla II. Se busca conocer el proceso 
de recogida de leche en la comarca, la variedad de productos derivados, su comercialización y el consumo de las familias del entorno. Se ha puesto un especial énfasis en comparar el valor nutricional de los diversos productos lácteos, para diferenciar entre los frescos, los elaborados industrialmente y los ecológicos. Este conocimiento permite decidir qué alimentos tomar de alto valor nutricional y precio bajo, frente a los procesados de alto coste.

Tabla II: Planificación del segundo plan de acción: la leche.

\begin{tabular}{|c|c|c|}
\hline TAREAS & SUBTAREAS & $\begin{array}{c}\text { ETAPA } \\
\text { EDUCATIVA }\end{array}$ \\
\hline $\begin{array}{c}\text { Preparación del } \\
\text { profesorado }\end{array}$ & Presentar el plan de acción al profesorado y trabajar sus objetivos. & El, EP y ESO. \\
\hline $\begin{array}{l}\text { Introducción } \\
\text { del programa al } \\
\text { alumnado }\end{array}$ & $\begin{array}{l}\text { 1. Estudiar el consumo de leche y sus derivados en su dieta. } \\
\text { Investigar qué tipo de leche y derivados consume el alumnado. } \\
\text { Mostrar la importancia en su dieta. } \\
\text { 2. Conocer, a través de visitas, diferentes modelos de producción } \\
\text { y distribución de leche: modelo sostenible y modelo de grandes } \\
\text { cadenas de distribución. }\end{array}$ & $\begin{array}{c}\operatorname{ESO}\left(1^{\circ}, 2^{\circ}, 3^{\circ}\right. \\
\left.\text { y } 4^{\circ}\right) .\end{array}$ \\
\hline $\begin{array}{c}\text { Diagnóstico } \\
\text { actual }\end{array}$ & $\begin{array}{l}\text { 1. Evaluar el valor nutricional de los tipos de leche y sus derivados } \\
\text { a través del estudio del etiquetado de los productos lácteos. } \\
\text { 2. Conocer la cantidad de leche y de productos lácteos que se } \\
\text { deberían consumir, desde un punto saludable. } \\
\text { 3. Estudiar y analizar las diferencias de un producto lácteo } \\
\text { elaborado industrialmente a otro que no. } \\
\text { 4. Comprender la diversidad de alergias a los productos lácteos, y } \\
\text { la inclusión de las personas que las padecen. } \\
\text { 5. Identificar si los diferentes envases de los productos lácteos } \\
\text { alteran o no la calidad del producto. }\end{array}$ & $\begin{array}{c}\text { EP }\left(5^{\circ} \text { y } 6^{\circ}\right) \\
\text { ESO }\left(1^{\circ}, 2^{\circ}, 3^{\circ}\right. \\
\left.\text { y } 4^{\circ}\right) .\end{array}$ \\
\hline Evolución & $\begin{array}{l}\text { 1. Analizar la importancia de la leche y derivados en la dieta } \\
\text { gallega, desde sus inicios hasta ahora. } \\
\text { 2. Conocer la evolución de los envases de los lácteos. } \\
\text { 3. Estudiar la evolución del etiquetado de los lácteos. }\end{array}$ & $\mathrm{ESO}\left(1^{\circ}\right.$ y $\left.2^{\circ}\right)$ \\
\hline Sostenibilidad & $\begin{array}{l}\text { 1. Realizar entrevistas a diferentes familias del centro para conocer } \\
\text { su consumo de productos lácteos. } \\
\text { 2. Analizar las entrevistas para valorar si se está realizando un } \\
\text { consumo responsable. } \\
\text { 3. Conocer productos lácteos ecológicos: dónde se venden, } \\
\text { quienes los pueden consumir, etc. } \\
\text { 4. Reflexionar sobre el destino de productos lácteos ecológicos. }\end{array}$ & $\begin{array}{c}\text { EP }\left(1^{\circ} \text { y } 2^{\circ}\right) \\
\text { ESO }\left(1^{\circ} \text { y } 2^{\circ}\right) .\end{array}$ \\
\hline Actividad final & $\begin{array}{l}\text { 1. Analizar la producción, calidad y cantidad de leche que pueden } \\
\text { producir las distintas razas de vacas de la comarca. } \\
\text { 2. Plasmar todos estos datos en una exposición abierta a toda la } \\
\text { comunidad educativa. }\end{array}$ & El, EP y ESO. \\
\hline
\end{tabular}

Fuente: Proyecto "Investigando la dimensión global de la educación para el desarrollo. Un proyecto piloto de investigación - acción colaborativa en un centro educativo de Galicia. Fase II" (PR 804A 2016/25). 


\section{Plan de acción 3: Mundo y sociedad}

Por último, el tercer plan (ver Tabla III) vincula los temas locales con los globales, a través de una reflexión sobre el tratamiento que la sociedad, y el mundo en general, hace de los alimentos, utilizando como instrumento la publicidad difundida por las multinacionales de la alimentación en los distintos medios de comunicación.

Sus objetivos específicos pretenden mostrar que la elección, y el consumo, están ligados a factores que nada tienen que ver con el valor nutricional. Se busca que identifiquen que esa publicidad incluye contenidos pseudocientíficos, que reconozcan la importancia de la imagen utilizada en los medios de comunicación y su influencia en el pensamiento social. En definitiva, que actúen de un modo crítico ante estos mensajes. La reflexión les permitirá establecer propuestas de actuación que contribuyan a minimizar las diferencias sociales planetarias y, finalmente, a fomentar la sensibilización por los problemas de otros pueblos ayudando segundo sus posibilidades. Como, por ejemplo, conocer la relación entre la deforestación de grandes espacios de selva y los grandes cultivos de soja, puesto que es una materia prima de aquellos productos que sustituyen con frecuencia a productos derivados de la leche.

Tabla III: Planificación del tercer plan de acción: mundo y sociedad.

\begin{tabular}{|c|c|c|}
\hline TAREAS & SUBTAREAS & $\begin{array}{l}\text { ETAPA } \\
\text { EDUCATIVA }\end{array}$ \\
\hline $\begin{array}{l}\text { Preparación del } \\
\text { profesorado }\end{array}$ & Presentar el plan de acción al profesorado y trabajar sus objetivos. & El, EP y ESO. \\
\hline $\begin{array}{l}\text { Introducción } \\
\text { del programa al } \\
\text { alumnado }\end{array}$ & $\begin{array}{l}\text { 1. Analizar los contenidos pseudocientíficos de la publicidad. } \\
\text { 2. Realizar un taller sobre el mundo publicitario. } \\
\text { 3. Crear un dossier sobre publicidad de productos lácteos: Investigar el público al } \\
\text { que va dirigido. Conocer diferentes medios de comunicación y cómo transmiten } \\
\text { la publicidad y en qué horario. Seleccionar una variedad de anuncios publicitarios } \\
\text { en diferentes formatos y épocas históricas. }\end{array}$ & $\begin{array}{l}\mathrm{ESO}\left(1^{\circ}, 3^{\circ}\right. \\
\left.\quad y 4^{\circ}\right)\end{array}$ \\
\hline $\begin{array}{l}\text { Diagnóstico } \\
\text { actual }\end{array}$ & $\begin{array}{l}\text { 1. Analizar publicidad audiovisual de productos lácteos : Revisar su contenido } \\
\text { científico, la imagen personal, el mensaje saludable, etc. Estudiar el tipo de } \\
\text { alimentación que trasmiten: } \\
\text { tradicional o no, con productos locales o no, etc. } \\
\text { 2. Comparar los anuncios publicitarios de diversos países. } \\
\text { 3. Estudiar cómo la publicidad informa de los conflictos. }\end{array}$ & ESO $\left(3^{\circ}\right.$ y $\left.4^{\circ}\right)$ \\
\hline Evolución & $\begin{array}{c}\text { 1. Buscar noticias relacionadas con la crisis del sector lácteo para conocer la } \\
\text { difusión del conflicto y la fuente. } \\
\text { 2. Estudiar cómo los anuncios publicitarios pueden generar formas de } \\
\text { discriminación, explotación infantil, etc. } \\
\text { 3. Revisar la influencia de las multinacionales del sector lácteo en la compra y } \\
\text { distribución de sus productos, y otros relacionados como el azúcar, el cacao, el } \\
\text { café, etc. } \\
\text { 4. Analizar las causas de la aparición del comercio justo. } \\
\begin{array}{c}\text { 5. Estudiar cómo la producción láctea puede generar problemas de justicia social, } \\
\text { e interdependencia planetaria. }\end{array}\end{array}$ & $\begin{array}{c}\text { EP }\left(2^{\circ}, 3^{\circ}, 4^{\circ}\right. \\
\left.5^{\circ} \text { y } 6^{\circ}\right) \\
\text { ESO }\left(1^{\circ}, 2^{\circ}\right. \\
\left.3^{\circ} \text { y } 4^{\circ}\right)\end{array}$ \\
\hline Sostenibilidad & $\begin{array}{l}\text { 1. Diseñar un anuncio publicitario para fomentar } \\
\text { los productos lácteos de la zona. } \\
\text { 2. Realizar un pequeño documental } \\
\text { sobre la situación del sector lácteo de la zona. }\end{array}$ & $\begin{array}{l}\text { ESO }\left(1^{\circ}, 2^{\circ}\right. \\
\left.3^{\circ} \text { y } 4^{\circ}\right)\end{array}$ \\
\hline Actividad final & $\begin{array}{c}\text { Realizar una campaña de concienciación sobre esta situación y proyectar una } \\
\text { imagen positiva del mundo rural. }\end{array}$ & El, EP y ESO. \\
\hline
\end{tabular}

Fuente: Proyecto "Investigando la dimensión global de la educación para el desarrollo. Un proyecto piloto de investigación - acción colaborativa en un centro educativo de Galicia. Fase II" (PR 804A 2016/25). 


\section{Reflexiones sobre un programa colaborativo e integral de ECG}

El desarrollo de este proceso nos ha permitido reflexionar sobre cómo incorporar la ECG a la escuela:

- El programa ilustrado en el presente artículo cumple las recomendaciones apuntadas por los especialistas para la incorporación de la ECG: el trabajo realizado en el centro ha sido integral, no actividades aisladas, y el programa ha emanado del grupo. Se ha fortalecido la colaboración constructiva y la cultura de centro, aportando experiencias al desarrollo colectivo y considerando a la institución como el núcleo del proyecto. Las interacciones se han ampliado al conectar en un único grupo de trabajo a los docentes del centro educativo, de Educación Infantil, Primaria y Secundaria, con el profesorado universitario y con miembros de una ONGD.

- Gracias al trabajo del profesorado, el programa presentado asocia todas las materias del curriculum, para un diseño integral y un tratamiento transversal de la temática. En este caso el adjetivo de colaborativo afecta a dos vertientes. Por un lado, se trata de enseñanza colaborativa, pues el profesorado diseña conjuntamente un programa de actividades para todas las materias, no sólo para las suyas. Por otro lado, las actividades están destinadas al aprendizaje colaborativo, en las que el alumnado trabaja en pequeños grupos para conseguir unos objetivos de aprendizaje común (Llorenç \& Forcano, 2014).

- La elección de un tema local permite que relacionen lo aprendido con su propia realidad a varios niveles: personal (nutrición, salud, consumo), ambiental (impacto del sector y conservación del medio), cultural (evolución histórica e influencia) y socioeconómico (desarrollo sostenible). La gran riqueza de este tipo de programación de ECG es que les faculta para usar ese conocimiento en la toma de decisiones sobre todos estos aspectos.

- Las estrategias didácticas utilizadas pretenden que el alumnado sea protagonista de su aprendizaje y favorecer el desarrollo de la competencia social y cívica: el debate, la relación de lo estudiado con la realidad del alumnado, el trabajo en grupo, la participación en el aula y las campañas de concienciación. Se busca que el centro educativo forme una ciudadanía activa, a través de la colaboración y el trabajo en grupo de la comunidad educativa, y preparada para la toma de decisiones sobre el medio que los rodea.

- La universidad ha sido clave como catalizadora de la interacción entre todos los niveles educativos, garantizando también la transversalidad y proponiendo una estructura de trabajo y programación aplicable en cualquier centro educativo. La creación en los planes de acción de esa estructura común de tareas (Preparación previa del profesorado, Introducción al alumnado, Diagnóstico, Evolución, Sostenibilidad y Síntesis) posibilita que este proceso de diseño del programa sea trasladable, a cualquier centro y a cualquier temática local de proyección global. 
- Se ha creado un programa a través del que participar y contribuir a la comunidad y a lo mundial (actuación de multinacionales de alimentación, explotación de recursos en los países, justicia social). En definitiva, una reflexión que permite confirmar el gran valor del uso de un tema local para proyectarlo a lo global.

Todo el proceso para incorporar la ECG al proyecto del centro ha supuesto un gran reto. La complejidad del número de docentes, su origen diverso y su formación académica dispar, así como la inclusión de factores relacionados con una educación "no formal" (procedentes de la experiencia de los miembros de la ONGD), han provocado más de un problema y retraso en el diseño. Pero también lo han enriquecido, de una forma tal que sólo podemos entender el programa resultante viendo la complementariedad que se ha conseguido entre todos los participantes.

Hemos aprendido que la necesidad de introducir la ECG en nuestros centros educativos, no pasa por aprender de experiencias de otros países, sino por desarrollar iniciativas propias que acerquen la temática a los implicados. Incorporarla, desde los primeros hasta los últimos niveles del sistema educativo, será la forma de abrir las puertas a la formación de un alumnado informado y capacitado para tomar decisiones.

\section{Referencias}

Aguado, G. (Coord.). (2011). Educación para el desarrollo y la ciudadanía global: guía para su integración en centros educativos. Madrid: Agencia Española de Cooperación Internacional para el Desarrollo.

Barkeley, E.F. (2007). Técnicas de aprendizaje colaborativo. Madrid: Morata.

Brownlie, A. (2001). Citizenship Education: the global dimension, guidance for key stages 3 and 4. London, UK: Development Education Association.

Davies, I., R., Evans, M \& Reid, A. (2005) Globalising citizenship education? A critique of "global education" and "citizenship education". British Journal of Educational Studies, 1, 66-89.

Davies, L. (2008). Global citizenship education. In Bajaj, M. (Ed.) Encyclopedia of Peace Education. Columbia: Teachers College, Columbia University.

Fricke, H.-J., Gathercole, C. \& Skinner, A. (2015). Monitoring education for global citizenship: a contribution to debate. DEEEP-CONCORD DARE Forum, European Development NGO confederation.

Imbernón, F. (2002). La investigación educativa y la formación del profesorado. In Imbernón, F. (Coord.). La investigación educativa como herramienta de formación del profesorado. Reflexión y experiencias de investigación educativa (pp. 11-68). Barcelona: Graó.

Kemmis, S. \& Mctaggart, R. (1988). Cómo planificar la investigación-acción. Barcelona: Laertes.

Leite, B. \& Beltrán, J. (2012). Universidad y sociedade: la pertinência de educación superior para una ciudadanía plena. Revista Lusófona de Educação, 21, 33-52.

Llorenç, J. \& Forcano, A. (2014). El aprendizaje colaborativo y las TIC en la clase de física. Alambique didáctica de las ciencias experimentales, 76, 38-44. 
Membiela, P. (2002). Investigación-acción en el desarrollo de proyectos curriculares innovadores de ciencias. Enseñanza de las Ciencias, 20 (3), 443-450.

Ortega, M. L. (2007). Estrategia de educación para el desarrollo de la cooperación española. Madrid: Ministerio de Asuntos Exteriores y de Cooperación.

Paz Abril, D. de (2007). Escuelas y educación para la ciudadanía global: una mirada transformadora. Barcelona: Intermón Oxfam.

Su, F., Bullivant, A. \& Holt, V. (2013). Global citizenship education. In Curtis, W., Ward, S., Sharp, J. \& Hankin, L. (Eds). Education studies: an issues based approach (3rd ed.). Exeter: Sage/Learning Matters.

Tawil, S. (2013). Education for "global citizenship": a framework for discussion. Unesco Working Papers, Education Research and Foresight.

Unesco (1974). Actas de la Conferencia General $18^{a}$ Reunión (Vol. 1), Resoluciones, París, 17 de octubre-23 de noviembre.

Unesco (2015). Educación para la ciudadanía mundial. Temas y objetivos de aprendizaje. Paris: Organización de las Naciones Unidas para la Educación, la Ciencia y la Cultura.

Unesco (2016). Educación para la ciudadanía mundial. Preparar a los educandos para los retos del siglo XXI. Paris: Organización de las Naciones Unidas para la Educación, la Ciencia y la Cultura.

Ánxela Bugallo-Rodríguez Doutora em Biologia pela Universidade de Santiago de Compostela (USC).

Professora da Universidade de A Corunha.

E-mail: anxela.bugallo@udc.es ORCID: https://orcid.org/0000-0002-8059-7083

María Cristina Naya-Riveiro Doutora em Matemática pela Universidade de Santiago de Compostela. Professora na Universidade de A Coruña.

E-mail: cristina.naya@udc.es ORCID: https://orcid.org/0000-0002-0584-3328

Correspondência Ánxela Bugallo-Rodríguez Universidad de Santiago de Compostela (USC), Facultad de Ciencias de la Educación, Campus Vida, S/N, 15782 Santiago de Compostela, A Coruña, España.

Data de submissão: Outubro 2017

Data de avaliação: Dezembro 2017

Data de publicação: Setembro 2018 